\title{
OPTIMIZING VoIP USING A CROSS LAYER CALL ADMISSION CONTROL SCHEME
}

\author{
Mumtaz AL-Mukhtar and Huda Abdulwahed \\ Department of Information Engineering, AL-Nahrain University, Baghdad, Iraq \\ almukhtarefulbrightmail.org
}

\begin{abstract}
Deploying wireless campus network becomes popular in many world universities for the services that are provided. However, it suffers from different issues such as low VoIP network capacity, network congestion effect on VoIP QoS and WLAN multi rate issue due to link adaptation technique. In this paper a cross layer call admission control (CCAC) scheme is proposed to reduce the effects of these problems on VoWLAN based on monitoring RTCPRR (Real Time Control Protocol Receiver Report) that provides the QoS level for VoIP and monitoring the MAC layer for any change in the data rate. If the QoS level degrades due to one of the aforementioned reasons, a considerable change in the packet size or the codec type will be the solution. A wireless campus network is simulated using OPNET 14.5 modeler and many scenarios are modeled to improve this proposed scheme.
\end{abstract}

\section{KEYWORDS}

VoIP Capacity, QoS, Cross-layering, VoWLAN, Codec Adaptation.

\section{INTRODUCTION}

Voice over IP (VoIP) has been widely used these years for its simplified infrastructure and significant cost savings. One of the most interesting use cases for VoIP is in combination with the IEEE 802.11 technology to provide wireless voice services to mobile devices such as laptops, smart phones and PDAs. The use of VoWLAN (VoIP over WLAN) makes it possible for mobile employee of an enterprise or a campus to be provided with cost effective voice and flexible services [1]. However, two technical problems need to be solved. The first is that the system capacity for voice can be quite low in WLAN. The second is that VoIP traffic and data traffic from traditional applications (web, e-mail, etc...) can interfere with each other and bring down VoIP performance.

Voice capacity is defined as the maximum number of voice sessions that can be supported simultaneously by a network under specific quality constraints [2]. Therefore, it is essential to determine the number of users a WLAN can support simultaneously without significantly degrading the QoS and to analyze the delay, jitter and packet loss of VoIP over WLAN.

The objective of this paper is to study the effect of different codecs in IEEE 802.11 multi-rate environment, and the VoIP packet payload size in order to develop a cross-layer call admission control scheme between MAC and application layers. This mainly aims to enhance the network capacity and quality for the VoIP calls with the accepted QoS constraints. This research focuses on addressing the congestion in the network and the multi-rate issue caused by the link adaptation technique in WLAN campus network.

The rest of the paper is organized as follows: section 2 introduces VoIP system, VoIP quality evaluation criteria, and related link adaptation concept. Section 3 provides a brief overview of several researches in the related area. Section 4 introduces the cross-layer call admission control DOI : $10.5121 /$ ijcnc. 2013.5410 
International Journal of Computer Networks \& Communications (IJCNC) Vol.5, No.4, July 2013

scheme. The simulation scenarios and results are presented in section 5. Finally, conclusion is drawn in section 6 .

\section{TECHNICAL BACKGROUND}

This section gives a VoWLAN overview inspecting VoIP quality constraints. Our focus in this research is to achieve better QoS under varying network conditions.

\subsection{VoIP System}

VoIP system consists of three essential components: codec, packetizer and playout buffer. At the sender side, the analog voice signals are converted to digital signals, compressed and then encoded by voice codecs. There are various voice codecs that are developed and standardized by the International Telecommunication Union - Telecommunication Sector (ITU-T), such as G.711, G.726, G.729, G.723.1a, etc. The subsequent process performed is packetization, where the encoded voice is fragmented into equal size of packets by the packetizer. Each packet generated is composed of the encoded voice and headers, which are added at different layers, specifically by layers of Real-time Transport Protocol (RTP), User Datagram Protocol (UDP), and Internet Protocol (IP), as well as by Data Link layer header. Moreover, RTP and Real-Time Control Protocol (RTCP) are designed at the application layer to support real-time applications [3].

The packets are sent out over IP network to its destination, where the reverse process of decoding and depacketizing of the received packets is carried out. Additionally, there are signaling protocols of VoIP namely Session Initiation Protocol (SIP) and H.323. These protocols establish VoIP calls. H.323 was standardized by ITU-T specifically to smoothly work together with PSTN. On the other hand, SIP was standardized by Internet Engineering Task Force (IETF) to support Internet applications, such as telephony. Figure 1 illustrates VoIP protocol stack with respect to its TCP/IP protocol standard [4].

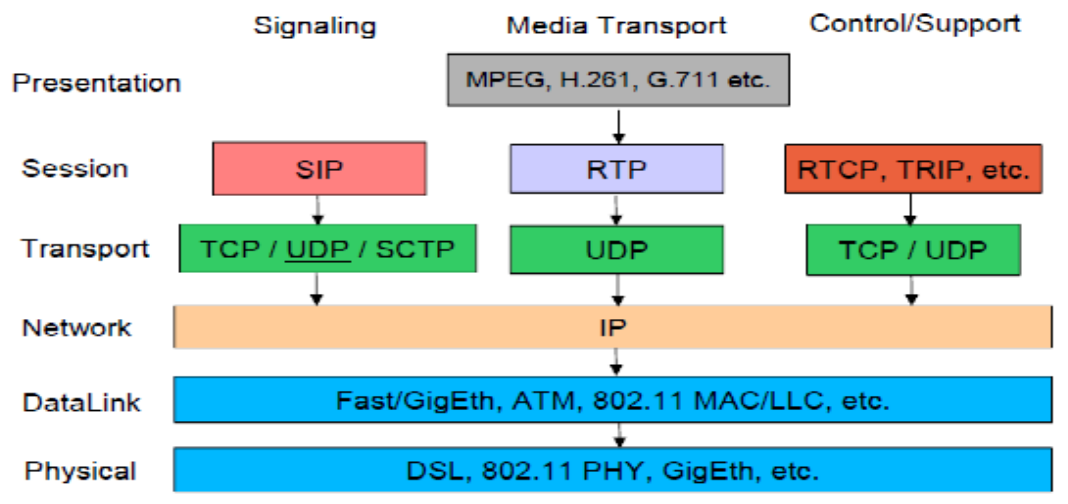

Figure 1. VoIP implementation in TCP/IP protocol standard

\subsection{VoIP QoS Evaluation}

The voice can be tested for quality in two ways, namely, subjective and objective. Humans perform the subjective voice testing by listening to the voice sample, whereas, objective tests are performed by computers. Common subjective benchmark for quantifying the performance of the speech codec is the Mean Opinion Score (MOS). For performing MOS test, a voice sample is given to a group of listeners. They listen to the sample and give a rating on a scale where 
"excellent" quality is given a score of 5 , "good" a 4, "fair" a 3, "poor" a 2, and "'bad" a 1. The ratings given by every member of the group is then averaged to get the MOS. The E-Model is most commonly used for objective measurements. The basic result of the E-Model is the calculation of the R-Factor. The R-factor is defined in terms of several parameters associated with a voice channel across a mixed Switched Circuit Network and a Packet Switched Network. The parameters included in the computation of the $\mathrm{R}$ factor are fairly extensive covering such factors as echo, background noise, signal loss, codec impairments, and others. R-factor can be expressed by (1) [5].

$$
R=R_{0}+I_{s}+I_{d}+I_{e}+A
$$

Where, $R_{0}$ groups the effects of noise, $I_{s}$ includes the effect of other impairments related to the quantization of the voice signal, $I_{d}$ represents the impairment caused by delay, Ie covers the impairments caused by low bit rate codecs and packet losses. The advantage factor $A$ compensates for the above impairments under various user conditions. For mobile telephony $A$ is assumed to be 10 . We consider that $\mathrm{A}$ is 0 in the case of VoIP.

MOS is related to R-Factor by (2) [2].

For $R<0: \quad M O S=1$

For $R>100: \quad M O S=4.5$

For $0<R<100: \quad M O S=1+0.035 R+7 \times 10^{-6} R(R-60)(100-R)$

The voice performance is considered acceptable if the end-to-end delay is less than $150 \mathrm{~ms}$ and the packet loss rate is less than $2 \%$ [6].

\subsection{IEEE 802.11 Standards and Link Adaptation}

A variety of wireless LAN technologies exist that use various frequencies, modulation techniques, and data rates. IEEE provides most of the standards, which are categorized as IEEE $802.11,802.11 a, 802.11 \mathrm{~b}, 802.11 \mathrm{~g}, 802.11 \mathrm{e}, \ldots .$. , and $802.11 \mathrm{n}$. For instance $802.11 \mathrm{~b}$ operates at 1,2 , 5.5, $11 \mathrm{Mbps}$, 802.11a \& 802.11g can support up to $54 \mathrm{Mbps}$ [7]. IEEE 802.11 supports the link adaptation technique, which allows a wireless transmitter to select an appropriate transmission rate on a packet basis according to the wireless link conditions. However, if a lower transmission rate is selected because of deterioration of the wireless link conditions, packet delays and losses will be increased due to the poor bandwidth availability, especially when the traffic load is high, and as a result, the quality of real-time applications will significantly deteriorate. The IEEE 802.11 standard does not specify how and when to switch between the permitted rates, so various link adaptation (LA) mechanisms have been studied [8]. One of the most effective solutions for this issue is to adapt the codecs of some of the active voice flows using codec adaptation algorithm (CAA) [9]. Cross layer methods have been designed to overcome numerous setbacks that were previously faced by wireless applications running on the strict layered OSI protocol stack. Cross layer design has numerous attributes. These include: fast stack adaptation, crossprotocol coordination, ease of QoS provisioning to different applications, prioritization ease, no unnecessary interaction between layers and improved throughput [10].

\section{RELATED WORK}

The two important issues related to VoWLAN are capacity and QoS depend on different parameters such as data rate, speech codec, delay, jitter and packet loss. Several techniques and algorithms have been presented to improve the quality of VoIP over WLAN. Cross-layer 
interaction approach has been introduced in several possible ways to optimize different layers' parameters. Papapanagiotoua et al. [11] exploit the interrelations between data rate, packetization interval, packet error rate, and retransmission attempts to produce a model to be used in the cross layer call admission control scheme in order to optimize QoS for single cell WLAN.

Several researches $[12,13,14,15,16]$ propose techniques to mitigate the effect of multi rate issues on VoIP quality over WLAN by changing either packet size or codec type depending on RTCP receiving messages and MAC layer data rate using either a cross layer control scheme or an adaptive algorithm. Luthra and Sharma [17] suggest VoWLAN QoS enhancement using numbers of QoS techniques like Integrated Services, Differentiated Services, and Resource Reservation Protocol. While Chakraborty et al. [18] introduce QoS upgrading by optimizing the concerned Access Points' parameters such as buffer size, retransmission limit, RTS threshold, transmission power, antenna type, location factors and network load. In [19] IEEE 802.11e standard has been harnessed to enhance the QoS compared with standard 802.11b/g.

The QoS for VoWLAN can be guaranteed using the smart call admission control scheme that is based on a dynamic bandwidth channel allocation to reserve the greater BW for VoIP application as introduced in [20]. However, authors in [21] and [22] invest for this purpose CAC (Call Admission Control) based on channel load estimation that is built around the TBIT (Time Between Idle Times) scheme. Jung et al. [23] employ call admission control scheme to achieve high link utilization via investigation of the on-off patterns of VoIP traffic from the Brady model.

Chang et al. [24] propose a QoS - aware path switching strategy by using stream control transmission protocol (SDCTP) in Multi-Protocol Label Switch (MPLS) network to improve VoIP traffic. The main contribution of this paper is that, the proposed CCAC scheme will enhance both the capacity and the QoS for VoWLAN in a campus-congested network based on two parameters: packet size and codec type.

\section{The proposed Cross layer Call Admission Control Scheme}

In order to improve the capacity and the QoS of VoIP over a WLAN, a cross layer call admission control scheme (CCAC) is proposed to allow the communication between MAC and application layers. This is depicted in figure 2 .

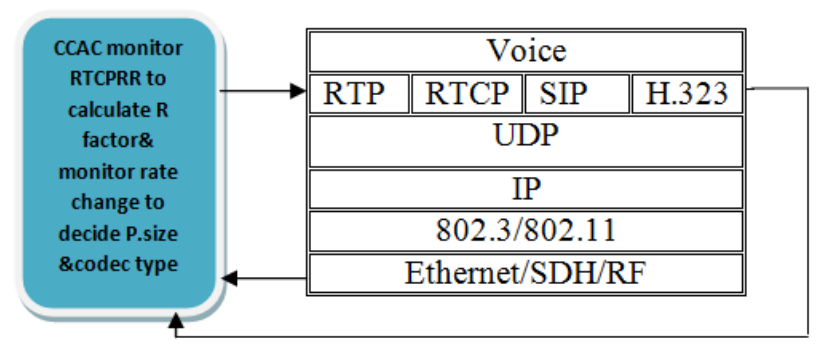

Figure 2. CCAC proposed scheme

This scheme tries to increase network VoIP capacity and to reduce the effect of two problems: the congestion and the WLAN link adaptation technique on VoIP calls. The periodic monitoring of the RTCPRR (Real Time Control Protocol Receiving Report) messages will be helpful to find if there is congestion in the network by evaluating the R-factor from the values of the end to end delay and packet loss ratio parameters of these messages. If these values are greater than the threshold values so that the end to end delay $>150 \mathrm{~ms}$ and packet loss ratio $>2 \%$ then the QoS for 
VoIP will be degraded indicating that $\mathrm{R}<70$ which is minimum value for the accepted QoS. In this case, the VoIP packet size will be checked whether it exceeds the predefined maximum size for the negotiated codec. In negative case the packet size will be increased by one frame and the monitoring process is resumed. However, if packet size exceeds the maximum then the codec will be changed to the one of a lower bit rate. Table 1 illustrates parameters of different codecs. If the transmission rate changes due to link adaptation algorithm then an alarm is send from the MAC Layer to the CCAC scheme which also decides to either adjust the packet size or change the codec type according to the RTCPRR. A descriptive flowchart of CCAC functions is shown in figure 3.

Table 1. CODEC Parameters

\begin{tabular}{|l|l|l|l|l|}
\hline \multicolumn{1}{|c|}{ CODEC Type } & \multicolumn{1}{c|}{ G.711 } & \multicolumn{1}{c|}{ G.726 } & \multicolumn{1}{c|}{ G.729A } & \multicolumn{1}{c|}{ G.723.1 } \\
\hline Bit rate kbps & 64 & 32 & 8 & 6.3 \\
\hline Bits per frame & 8 & 4 & 80 & 159 \\
\hline Algorithmic delay (ms) & 0.125 & 0.125 & 15 & 37.5 \\
\hline Codec delay (ms) & 0.25 & 0.25 & 25 & 67.5 \\
\hline Compression type & PCM & ADPCM & CSACELP & ACELP \\
\hline Complexity (MIPS) & $<<1$ & $\approx 1$ & $<=11$ & $<=18$ \\
\hline MOS & 4.1 & 3.85 & 3.7 & 3.6 \\
\hline
\end{tabular}


International Journal of Computer Networks \& Communications (IJCNC) Vol.5, No.4, July 2013

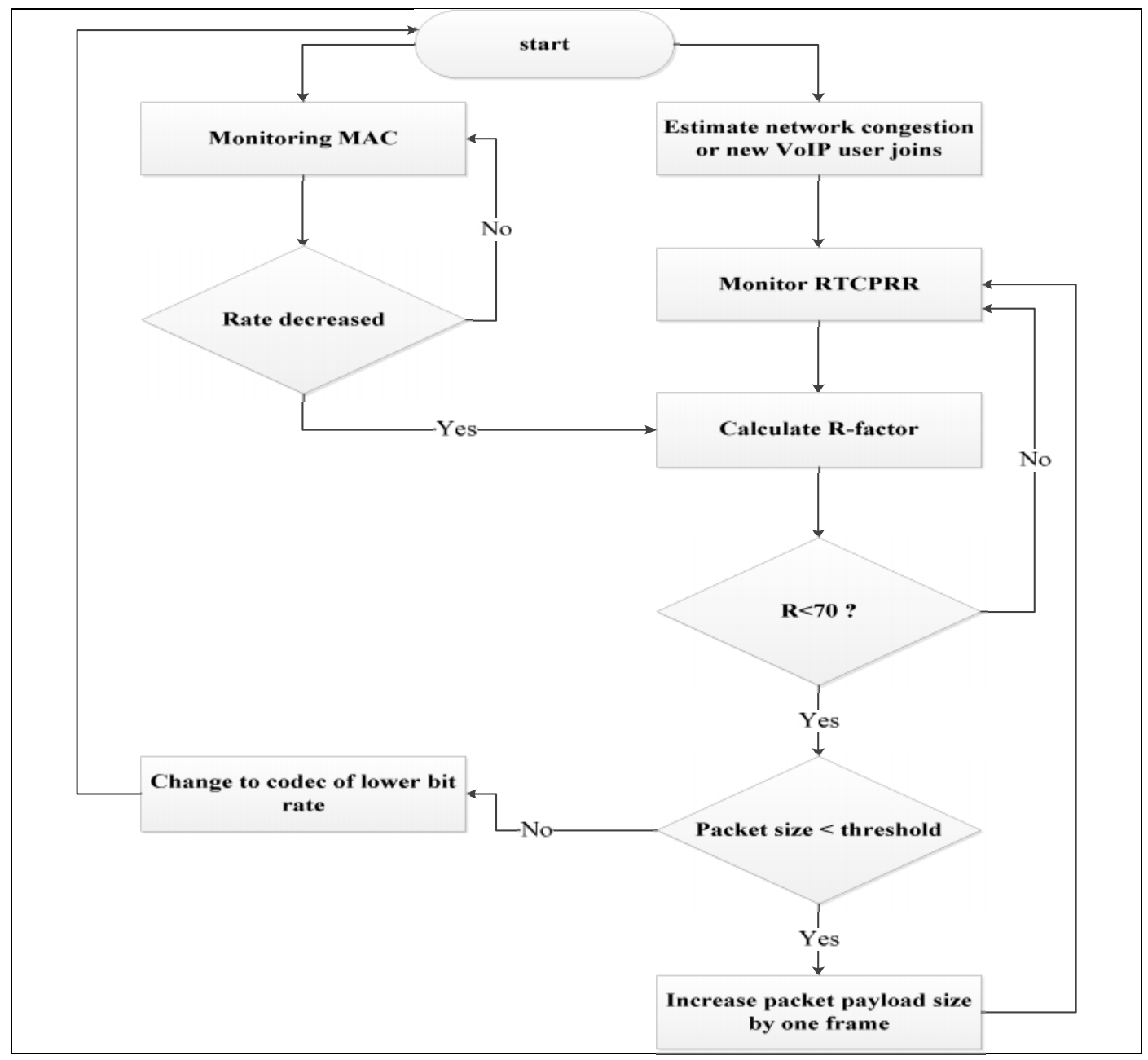

Figure 3. CCAC Flow chart

\section{Simulation \& RESUltS}

To assess how the proposed CCAC scheme can enhance the network performance in different conditions, the university wireless campus network has been simulated using OPNET modeler 14.5 [25]. Many scenarios have been imposed using different packet size, data rate, and different codecs to cover all possible cases.

\subsection{Simulation Setup}

The simulated wireless campus network consists of seven buildings; each one has three WLAN subnets with two servers (FTP\& DB) and a separate subnet of three wireless servers dedicated for Email, Heavy browsing \& Web searching applications. In addition there is an SIP sever for calls management. This network is connected to the Internet as shown in figures 4, 5, and 6 . 
International Journal of Computer Networks \& Communications (IJCNC) Vol.5, No.4, July 2013

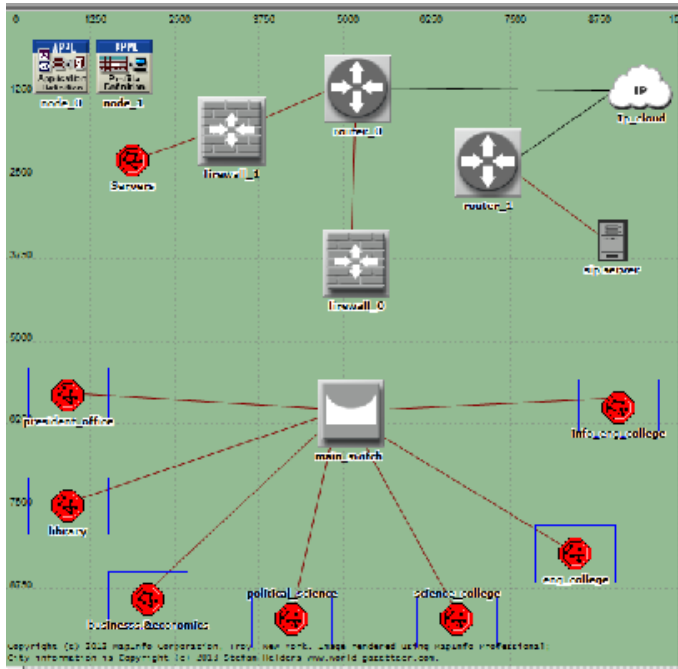

Figure 4. Campus Network

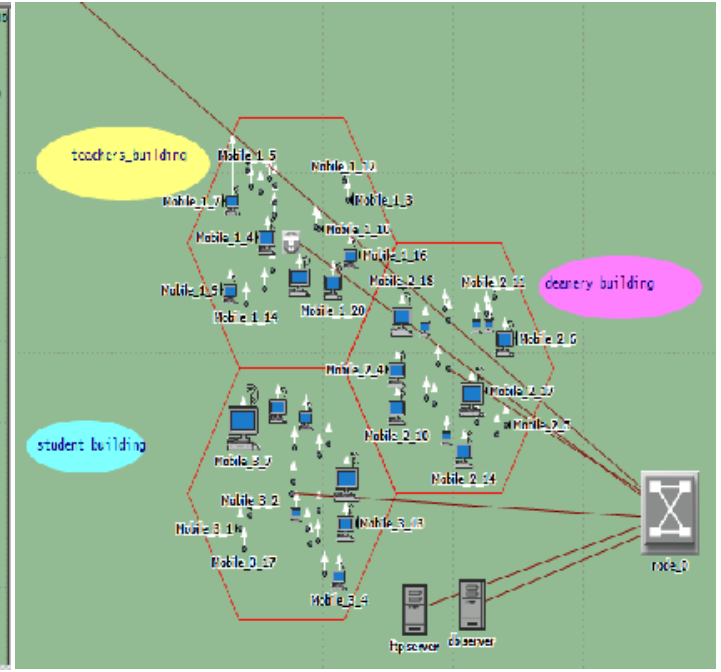

Figure 5. Building Subnet

Each WLAN subnet has 20 wireless work-station mobile nodes communicating with each other through an Access Point. The nodes are using IEEE802.11b standard for their communication with transmission rate of $11 \mathrm{Mbps}$. Six applications are defined in this network which are VoIP, FTP, DB, Email, web searching, and heavy browsing. The codec chosen for VoIP is G.711 with one frame per packet. The VoIP call is assumed to be between two nodes, caller and the callee.

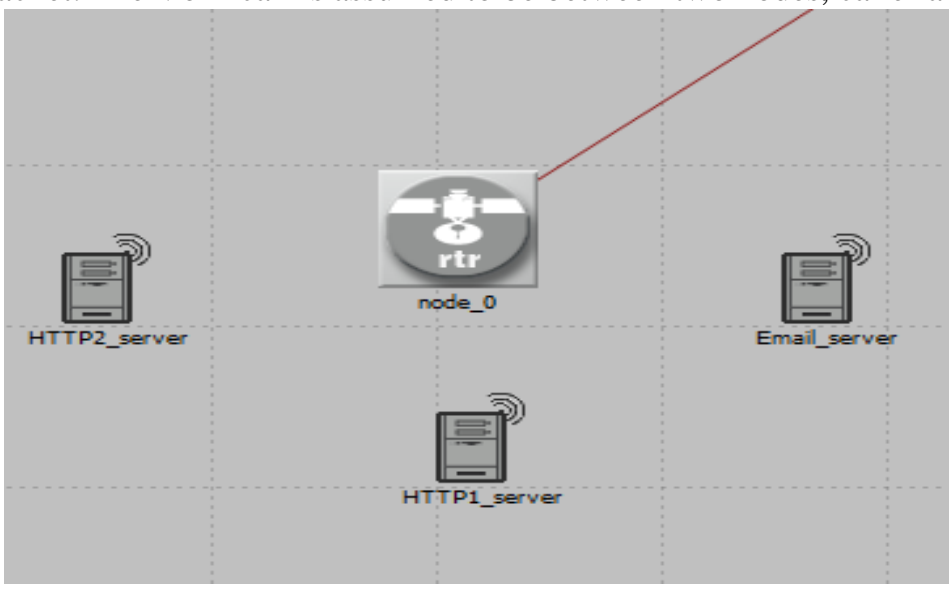

Figure 6. Server's Subnet

\subsection{Simulation Scenarios and Results}

Simulation scenarios cover two main cases as follows:

\subsubsection{Effects of Congestion and Addition of New VoIP User}

The first scenario aims to find how many simultaneous calls can be achieved in the network with packet size of one frame and G.711codec for VoIP application. Only five calls can be found in the same time so that the required end to end delay, MOS, and packet loss will be acceptable. Adding new calls or other traffic cause the performance to be unacceptable. These results are depicted in 
International Journal of Computer Networks \& Communications (IJCNC) Vol.5, No.4, July 2013

figures 7 and 8. In the second scenario, the VoIP packet size has been increased to comprise two frames. In this scenario, 10 simultaneous VoIP calls can be found and other traffic could be added. In the third scenario, the packet size has been increased to 3 frames to achieve 14 calls with more added traffic. In the fourth scenario the number of frames (F) considered is 4 and the simultaneous calls $(\mathrm{N})$ are 18. In the fifth scenario $\mathrm{F}=5$ and $\mathrm{N}=64$ with each node in the network has at least three running application so that the network is congested. The MOS is found to be $>3$, end to end delay $<150 \mathrm{~s}$ and packet loss ratio $<2 \%$ which are accepted. Adding more frames to the VoIP packet of the G.711 codec will produce unacceptable results. Figures 9a\& b show the maximum number of $\mathrm{N}$ and MOS values with respect to $\mathrm{F}$. Next scenario is considered when there is a new call, a codec change for this call to G.726 of $32 \mathrm{Kbps}$ with 2 frames per packet will occur for this call to be accepted.

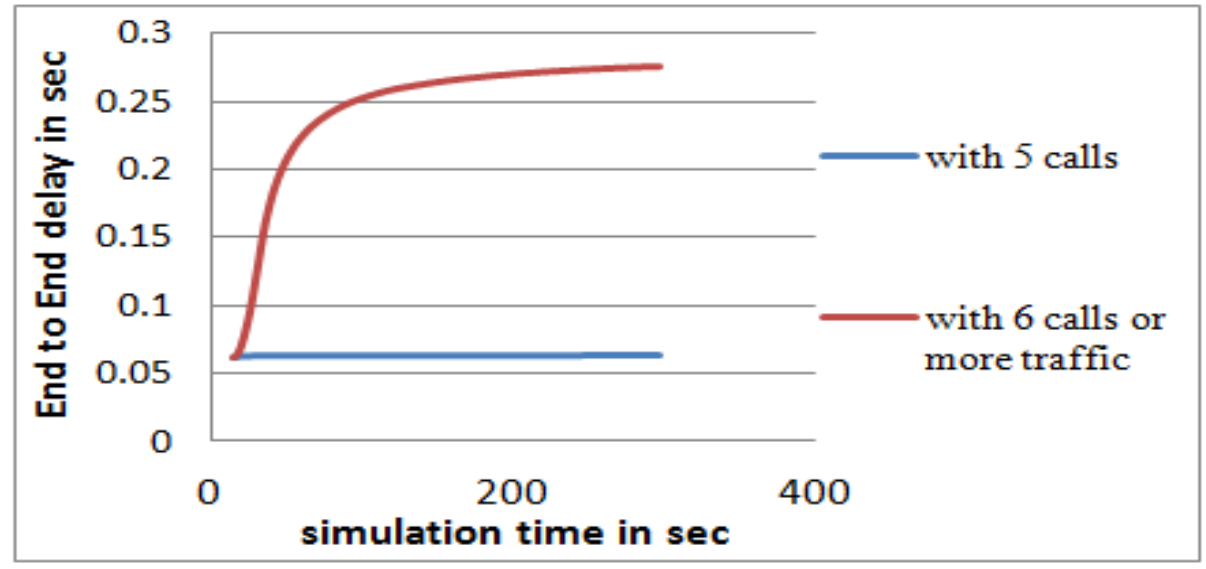

Figure 7. End to End Delay (case1, scenario1)

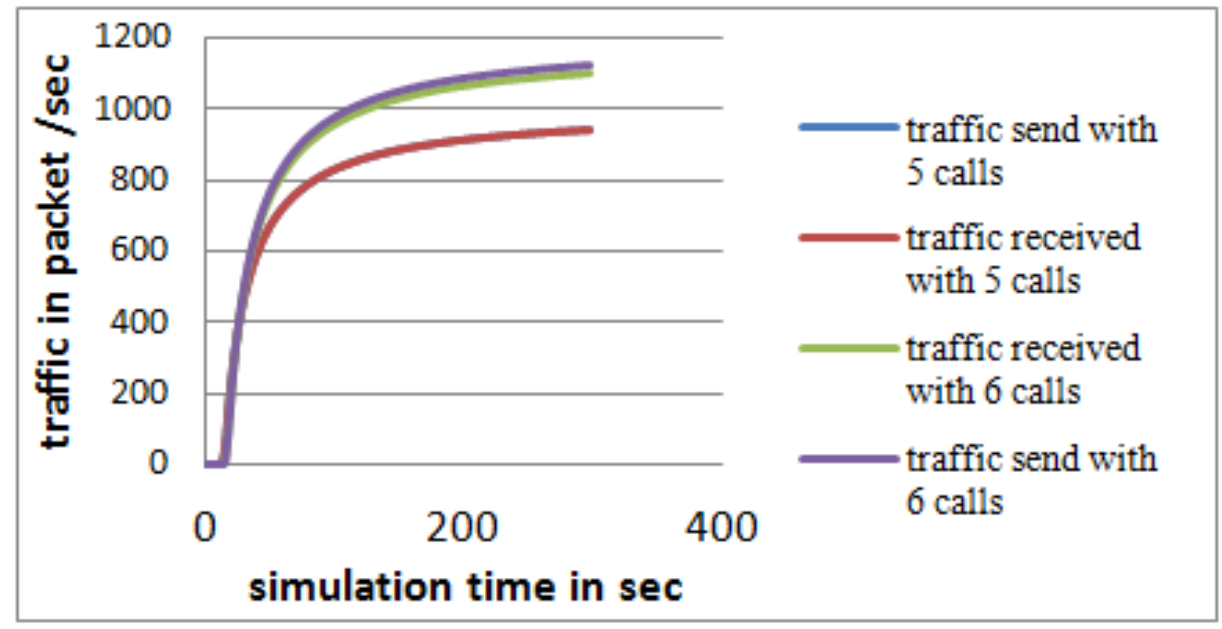

Figure 8. Traffic send and Traffic received (case1, scenario1) 
International Journal of Computer Networks \& Communications (IJCNC) Vol.5, No.4, July 2013

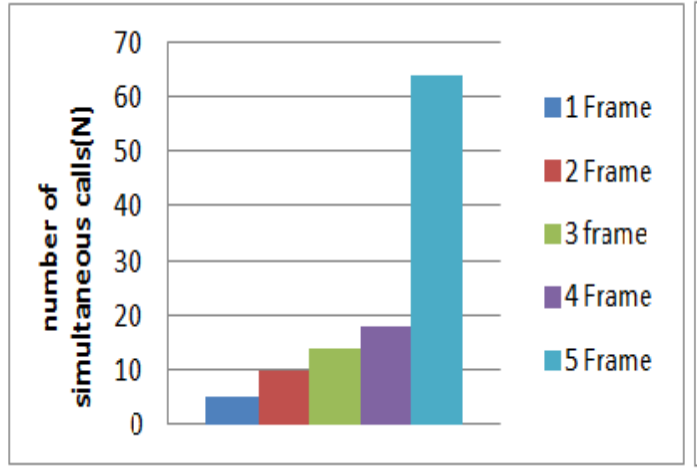

Figure 9a. Max. $\mathrm{N}$ with respect to $\mathrm{F}$

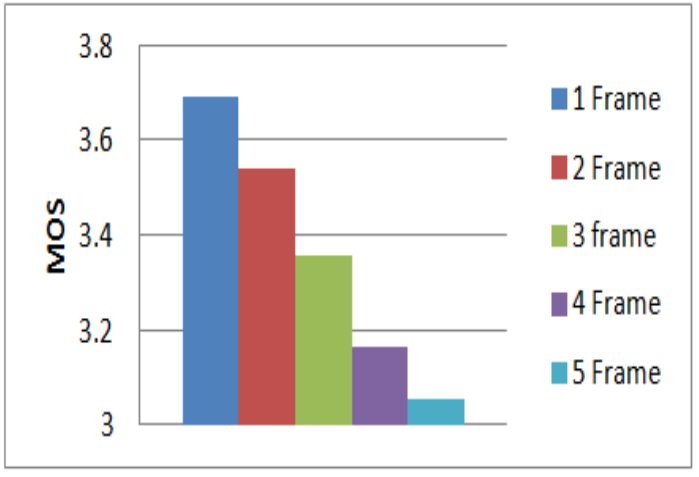

Figure 9b. MOS value with respect to F

\subsubsection{Effect of Multi Rate Issue}

Many scenarios have been introduced to cover changes in transmission rate from 11 to 5.5, 2, and $1 \mathrm{Mbps}$ for certain VoIP nodes. The packet size has been varied from 1 to 5 frames and the codec deployed is G.711. In the first scenario, the VoIP packet contains one frame and the transmission rate varies to $5.5 \mathrm{Mbps}$. Only the end-to-end delay will be affected but it is still accepted. Nevertheless, when a rate of $2 \& 1 \mathrm{Mbps}$ is deployed, the performance will be unacceptable. By increasing the packet size to 2 frames the performance is enhanced as shown in figures 10 and 11.

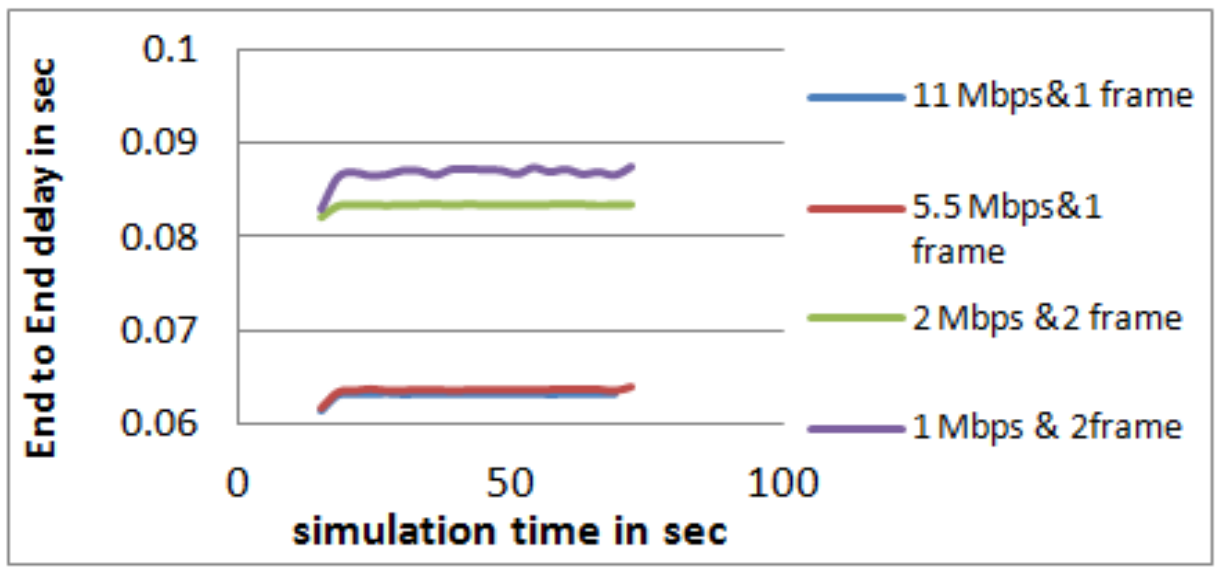

Figure 10. End to End Delay (case 2, scenario 2)

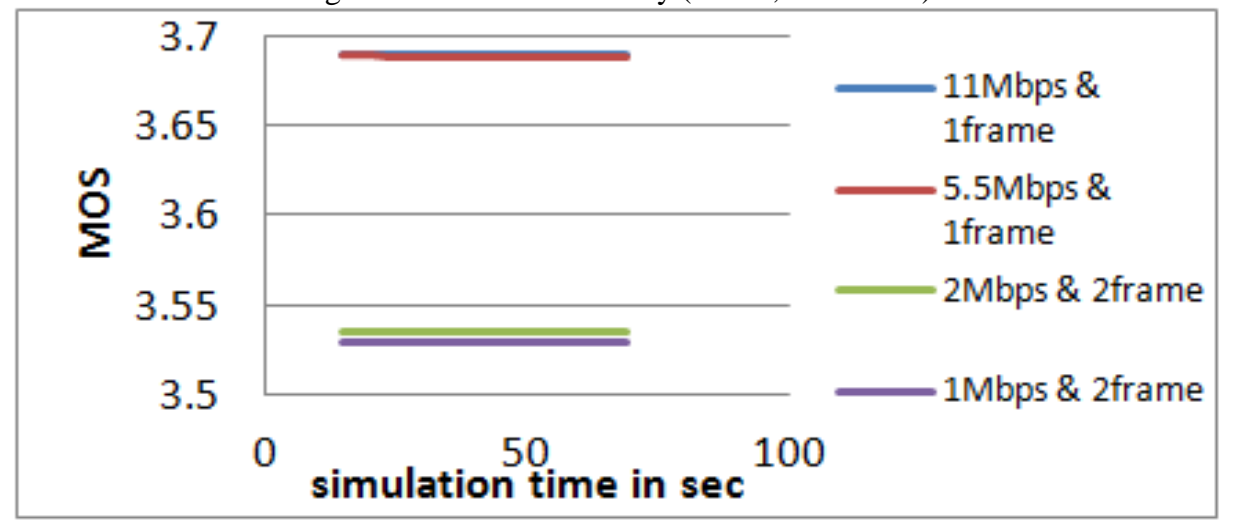

Figure 11. MOS value (case 2, scenario 1) 
International Journal of Computer Networks \& Communications (IJCNC) Vol.5, No.4, July 2013

The second scenario is considered when the VoIP node's transmission rate is $11 \mathrm{Mbps}$ but the packet size is 2 frames and a transmission rate changes within 5.5, 2, and $1 \mathrm{Mbps}$. A change in the packet size is made to enhance the VoIP quality of service parameters so it can be accepted. This is shown in figures $12 \& 13$. When the transmission rate is $2 \mathrm{Mbps}$ and the frame size is 2 frames the end to end delay is not acceptable i.e. > $150 \mathrm{~ms}$. However, at a packet size of 3 frames the end to end delay will be acceptable.

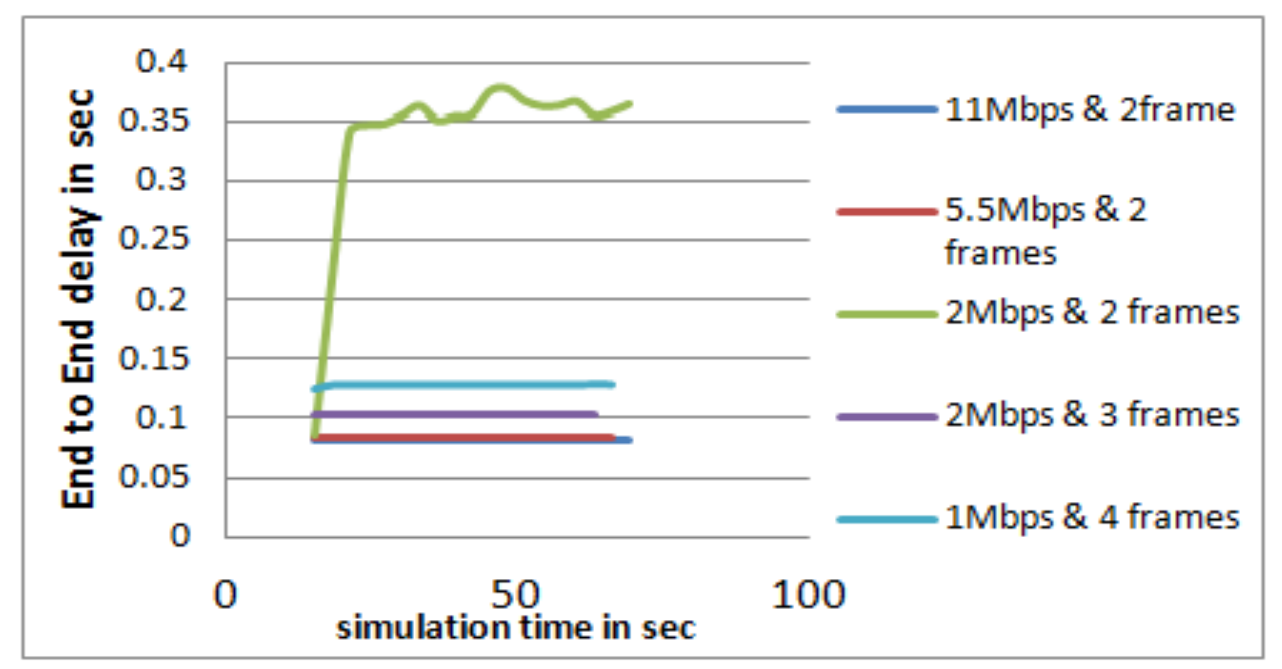

Figure 12. End to End Delay (case 2, scenario2)

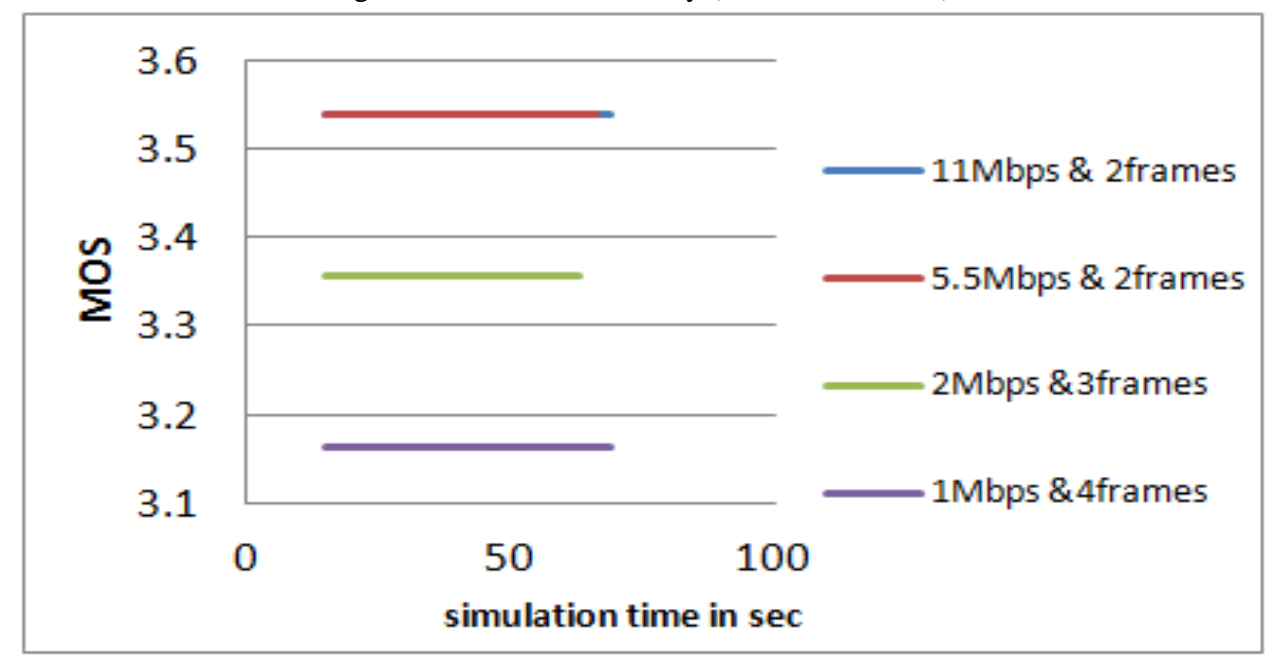

Figure 13. MOS value (case 2, scenario 2)

The third scenario is considered when VoIP packet size is 3 frames at transmission rate of 11 Mbps and it varies to 5.5, 2, and 1Mbps. At 5.5\& $2 \mathrm{Mbps}$, the packet size changes to 4 frames to get the accepted QoS parameters values. At $1 \mathrm{Mbps}$ increasing the packet size will not enhance the performance so the codec type will be switched to G.726 with packet size of 4 frames as shown in figures $14 \& 15$. 


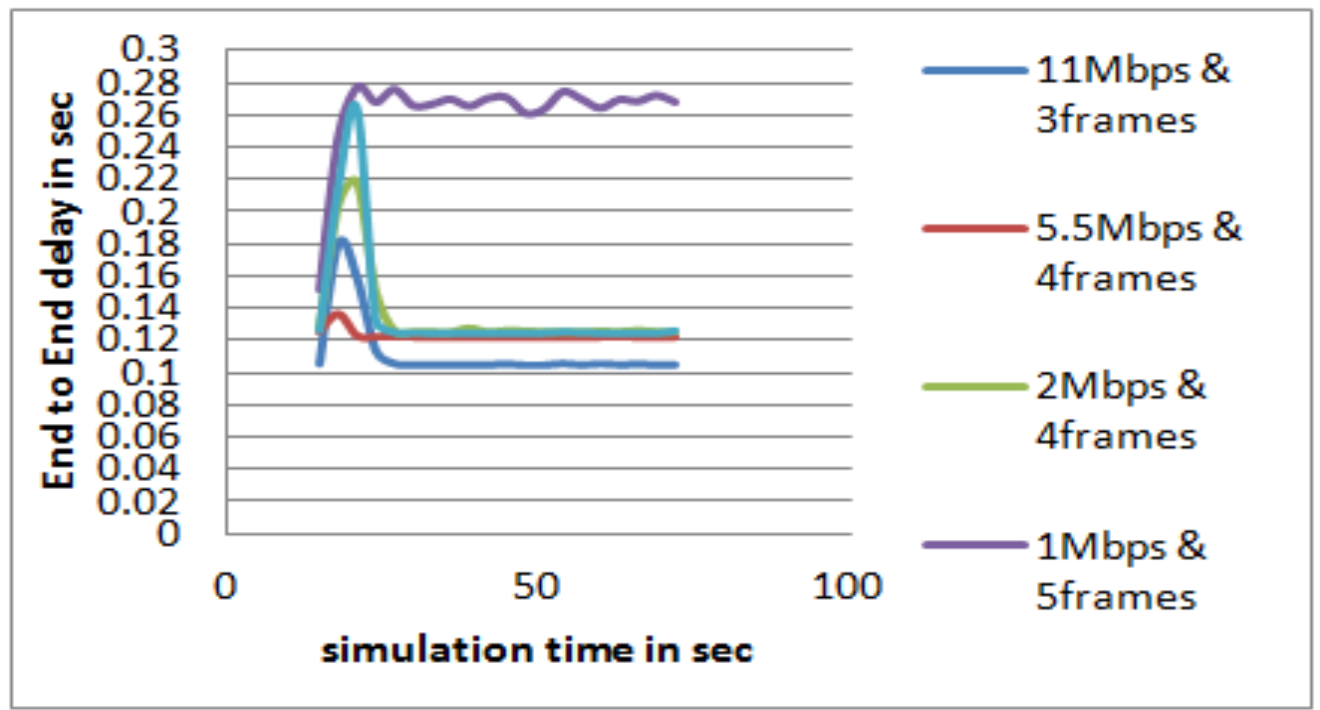

Figure 14. End to End delay (case 2, scenario 3)

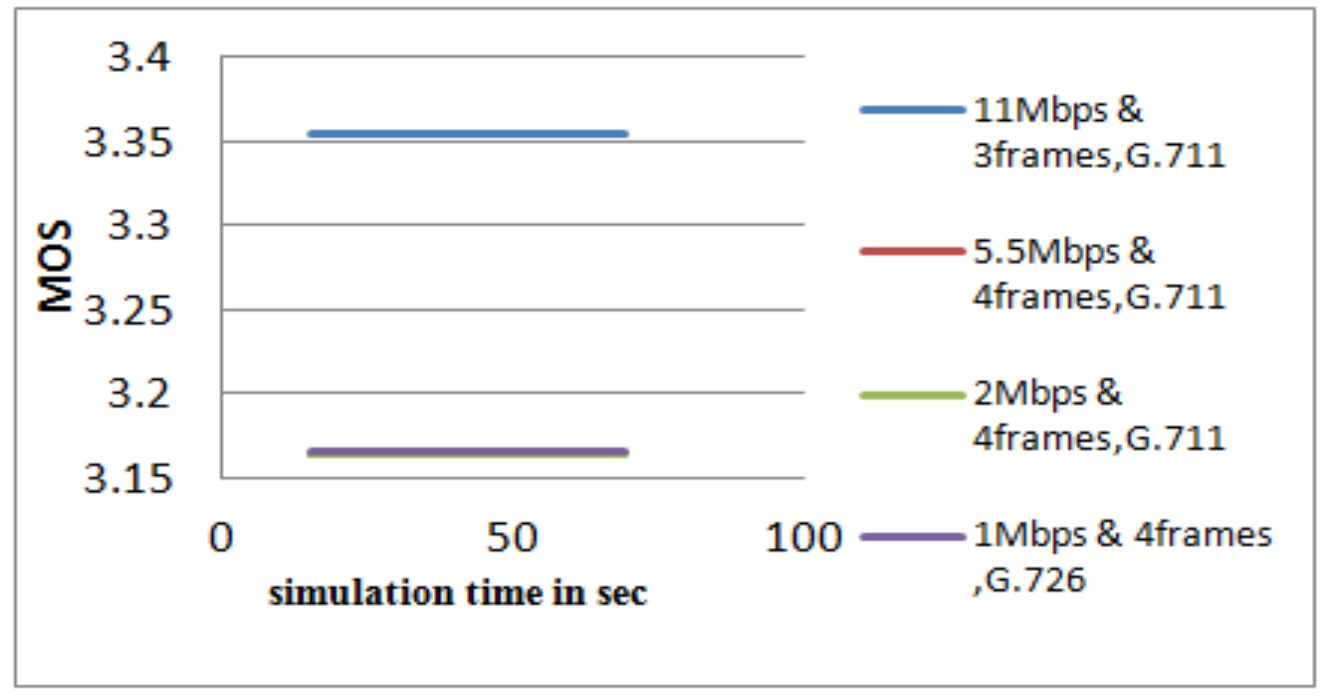

Figure 15. MOS value (case 2, scenario 3)

The fourth scenario is considered when the VoIP packet size consists of 4 frames and some VoIP nodes change their transmission rate from $11 \mathrm{Mbps}$ to 5.5, 2, $1 \mathrm{Mbps}$. At 5.5Mbps changing the codec will be more effective than packet size so G.726 will be the codec. At 2 Mbps also the codec will be changed to the one of a lower bit rate which is G.729 A. That is even with 5 frames packet size of the G.726 codec the quality would not be acceptable. At 1Mbps G.729A with 5 VoIP frames also does not provide good quality so it is replaced with G.723.1 of $6.3 \mathrm{Kbps}$ so the calls could be continued. These results are depicted in figures $16 \& 17$. 


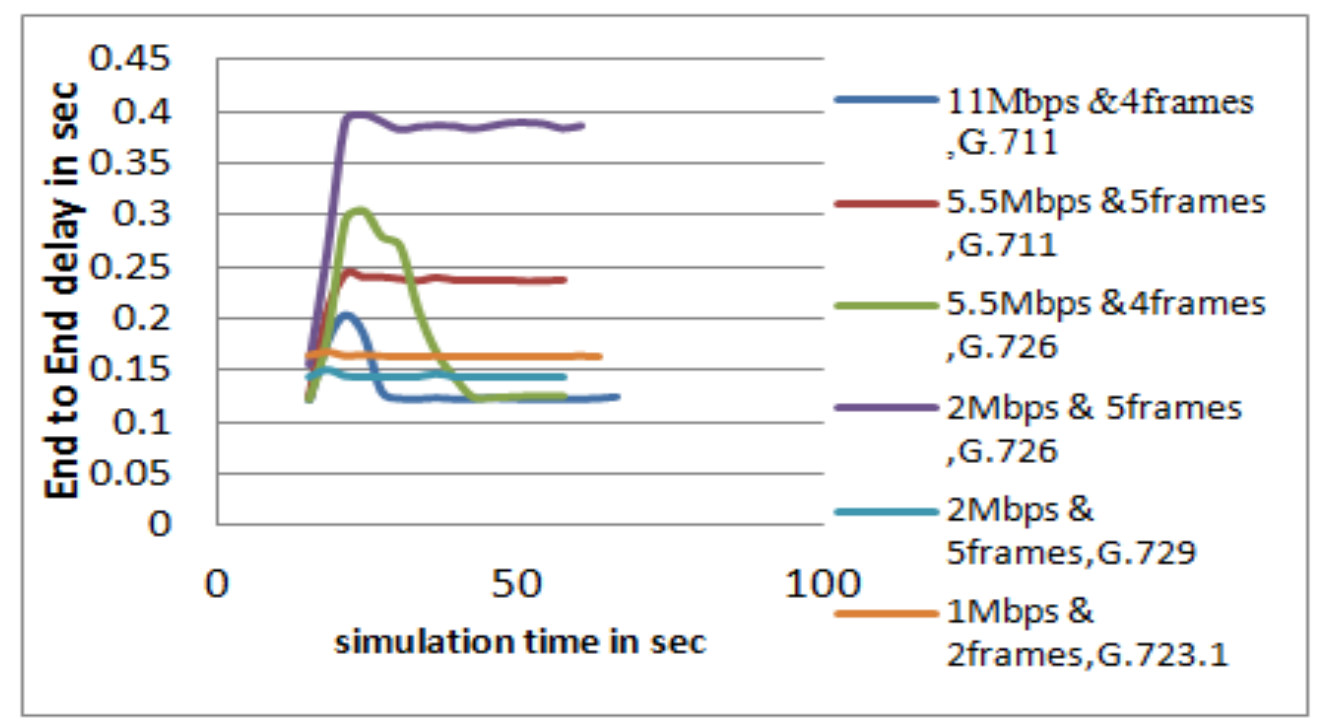

Figure 16. End to End delay (case 2, scenario 4)

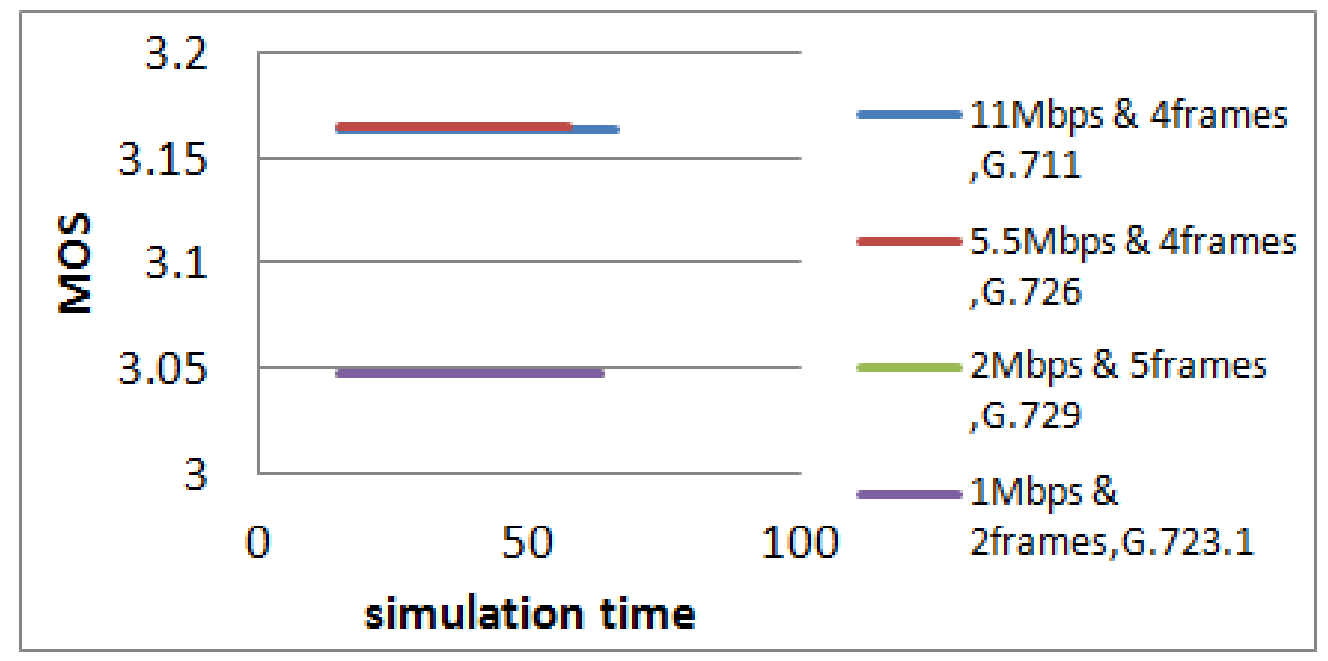

\section{CONCLuSion}

Figure 17. MOS value (case 2, scenario 4)

In this paper, the VoWLAN application is tested for performance in campus network. A cross layer call admission control scheme is proposed to enhance network capacity and QoS for VoIP by adapting packet size or codec type. The simulation results proved that the proposed CCAC scheme provides an efficient and fast way for solving the network congestion effect on VoIP application. Increasing network VoIP capacity and reducing the multi rate effect is achieved by monitoring the RTCPRR for the last end to end delay and Packet loss ratio that provides the network QoS level, which is represented by R-factor. As well as the MAC layer is monitored for any changes in the data rate of the mobile node. If the QoS degrades due to one of the above problems then the solution will be by either varying the packet size or codec type to optimize network performance without dropping the call. 
International Journal of Computer Networks \& Communications (IJCNC) Vol.5, No.4, July 2013

\section{REFERENCES}

[1] X. Wei, Y. Bouslimani, \& K. Sellal, (2012) "VoIP Based Solution for the Use over a Campus Environment" 25th IEEE Canadian Conference on Electrical and Computer Engineering (CCECE), PP 1-5.

[2] C. Brouzioutis , V. Vitsas \& P. Chatzimisios, (2010) "Studying the Impact of Data Traffic on Voice Capacity in IEEE 802.11 WLANs", IEEE International Conference on Communications, PP $1-6$.

[3] M. N. Ismail, (2010) "Analysis of Secure Real Time Transport Protocol on VoIP over Wireless LAN in Campus Environment" International Journal on Computer Science and Engineering (IJCSE), Volume 02, No. 03, PP 898-902.

[4] S. A. A. Alshakhsi \& H. Hasbullah, (2010) "Improving QoS of VoWLAN via Cross-Layer Interaction Approach", International Symposium on Information Technology, PP 678-682.

[5] A. Mukhopadhyay, T. Chakraborty, S. Bhunia, I. Misra \& S. Sanyal, (2011) "Study of Enhanced VoIP Performance under Congested Wireless Network Scenarios", Third International Conference on Communication Systems and Networks (COMSNETS 2011), PP 1-7.

[6] W. Wang, S. Liew, \& V. Li, (2005) "Solutions to Performance Problems in VoIP Over a 802.11 Wireless LAN", IEEE Transactions on Vehicular Technology journal, Volume 54, Issue 1, PP 366-384.

[7] D. E. COMER, (2008) Computer Networks and Internets, Pearson Education, Inc., Fifth Edition.

[8] T. Kawata, \& H. Yamada, (2006) "Adaptive Multi-Rate VoIP for IEEE 802.11 Wireless Networks with Link Adaptation Function”, Proceedings of the Global Telecommunications Conference (GLOBECOM '06), PP 1-5.

[9] Z. Chen, L. Wang, F. Zhang, X. Wang \& W. Chen, (2008) "VoIP over WLANs by Adapting Transmitting Interval and Call Admission Control", IEEE International Conference on Communications, PP 3242-3246.

[10] V. Sentongo \& H. Chan, (2009) "Optimization of Quality of Service Requirements for Real-Time Applications using Cross Layer Design”, IEEE AFRICON'09, PP 1-9.

[11] I. Papapanagiotoua, F. Granellib, D. Kliazovichb, \& M. Devetsikiotis, (2011) "A Metamodeling Approach for Cross-Layer Optimization: A Framework and Application to Voice over WiFi", Simulation Modelling Practice and Theory Journal, Volume 19, Issue 9, PP 2117-2129.

[12] S. Ali Alshakhsi, \& H. Hasbullah, (2011) "Improving QoS of VoWLAN via Cross-Layer-Based Adaptive Approach", International Conference on Information Science and Applications, PP 1-8.

[13] M. Tuysus \& H. Mantar, (2010) "Evaluation of Cross Layer QoS Approach for Improving Voice Quality over Multi Rate WLANs", International Conference on Computer Engineering and Systems (ICCES), PP 73-78.

[14] A. Sfairopoulou, B. Bellalta, C. Macian, \& C. Oliver, (2011) "A Comparative Survey of Adaptive Codec Solutions for VoIP over Multirate WLANs: A Capacity versus Quality Performance TradeOff" EURASIP Journal on Wireless Communications and Networking, Volume 2011, Issue 1, PP $1-13$.

[15] H. Kazemitabar \& A. Saida, (2011) "An Adaptive Rate Control Algorithm for VoIP over MultiRate WLANs", 2nd World Conference on Information Technology (WCIT-2011), PP 1087-1092.

[16] P. McGovern, P. Perry \& S. Murphy, (2011) "Endpoint-Based Call Admission Control and Resource Management for VoWLAN", IEEE Transactions on Mobile Computing, Volume 10, NO. 5, PP 684-699.

[17] P. Luthra \& M. Sharma, (2012) "Performance Evaluation of Audio Codecs using VoIP Traffic in Wireless LAN using RSVP”, International Journal of Computer Applications, Volume 40, No.7, PP 15-21.

[18] T. Chakraborty, A. Mukhopadhyay, S. Bhunia, I. S. Misra \& S. K. Sanyal, (2012) "An Optimization Technique for Improved VoIP Performance over Wireless LAN", Journal of Networks, Volume 7, NO. 3, PP 480-493.

[19] S. V. Bhanu \& R. M. Chandrasekaran, (2012) "Voice Call Capacity Analysis and Enhancement of IEEE 802.11 WLAN”, European Journal of Scientific Research, Volume 76, No.2, PP 271-280.

[20] R. Ganiga, B. Muniyal \& Pradeep, (2012) "Characteristic Analysis of VoIP Traffic for Wireless Networks In Comparison with CBR using QualNet Network Simulator” International Journal of Computer Applications, Volume 50, No.11, PP 25-31. 
International Journal of Computer Networks \& Communications (IJCNC) Vol.5, No.4, July 2013

[21] K. Yasukawa, A. G. Forte \& H. Schulzrinne, (2007) "Distributed Delay Estimation and Call Admission Control in IEEE 802.11 WLANs", IEEE International Conference on Network Protocols, PP 334-335.

[22] P. Dini, N. Baldo \& J. Nin-Guerrero, (2010) "Distributed Call Admission Control for VoIP over 802.11 WLANs based on Channel Load Estimation" IEEE International Conference on Communications journal, PP 1-6.

[23] Jung Ji-Young , Seo Dong-Yoon \& Lee Jung-Ryun, (2013) "VoIP Call Admission Control Scheme Considering Voip on-off Patterns", International Conference on Information Networking (ICOIN), PP 371 - 374.

[24] Chang Lin-huang, Lee Tsung-Han, Chu Hung-Chi, Lo Yu-Lung \& Chen Yu-Jen, (2013) "QoSAware Path Switching for VoIP Traffic using SCTP", Computer Standards \& Interfaces Journal, Volume 35, Issue 1, PP 158-169.

[25] A. S. Sethi \& V.Y. Hnatyshin, (2013) The Practical OPNET User Guide for Computer Network Simulation, CRC Press. 